\title{
Isotermas de adsorção e calor latente de vaporização de grãos de milho
}

\author{
Adsorption isotherms and vaporization latent heat of corn
}

\section{Samuel Gonçalves Ferreira Dos SANTOS ${ }^{1}$; Hellismar Wakson da SILVA²; Juliano Silva QUEIROZ; Renato Souza RODOVALHO4; Valdoméria Neves de Moraes MORGADO 5}

${ }^{1}$ Autor para correspondência, Acadêmico do curso de Agronomia, Instituto Federal Goiano - Campus Ceres, samuel-2100@hotmail.com

2 Engenheiro Agrônomo, Doutorando em Agronomia/Fitotecnia, Universidade Federal de Lavras - UFLA, Lavras-MG, email: waksonhellismar@gmail.com

${ }^{3}$ Acadêmico do curso de Agronomia, Instituto Federal Goiano - Campus Ceres, julianoagro260@gmail.com

${ }^{4}$ Doutor em Agronomia, IF Goiano - Campus Ceres, renato.rodovalho@ifgoiano.edu.br

${ }^{5}$ Doutora em Linguística, IF Goiano - Campus Ceres, valdomeria.morgado@ifgoiano.edu.br

Recebido em: 29-08-2018; Aceito em: 10-09-2019

\begin{abstract}
Resumo
As isotermas de adsorção podem ser definidas como a relação entre a atividade de água e umidade do grão em temperatura específica e servem como parâmetros para estabelecer condições adequadas de temperatura e umidade relativa de armazenagem. $\mathrm{O}$ objetivo deste trabalho foi determinar e modelar as isotermas de adsorção dos grãos de milho nas temperaturas do ar de 40,50 e $60 \stackrel{\circ}{\circ}$ e atividade de água entre 0,11 e 0,81 , bem como determinar o calor latente de vaporização da água. Para isso, os experimentos de sorção foram realizados pelo método estático gravimétrico em câmara tipo BOD, com o uso de soluções salinas saturadas. Diversos modelos matemáticos foram ajustados aos dados experimentais e a seleção do melhor modelo foi realizada por critérios estatísticos. O modelo Henderson Modificado é o que melhor representa as isotermas de adsorção dos grãos de milho nas condições estudadas. Os valores do calor latente de vaporização dos grãos de milho, com teores de água de 0,026 a 0,172 (b.s.), variaram de 3030,22 a $2567,92 \mathrm{~kJ} \mathrm{~kg}^{-1}$. O aumento da umidade nos grãos acarreta menores valores de calor latente de vaporização.
\end{abstract}

Palavras-chave adicionais: água; energia; equilíbrio higroscópico; Zea mays.

\begin{abstract}
The adsorption isotherms can be defined as the relationship between water activity and moisture content of the grain at a specific temperature and serve as parameters to establish appropriate storage temperature and relative humidity conditions. The objective of the present work was to determine and model the adsorption isotherms of corn kernels at air temperatures of 40,50 and $60{ }^{\circ} \mathrm{C}$ and water activity between 0.11 and 0.81 , as well as determine the latent heat of water vaporization. For this, the sorption experiments were carried out by the static gravimetric method in a BOD chamber, using saturated salt solutions. Several mathematical models were fitted to the experimental data and the selection of the best model was performed by statistical criteria. The Modified Henderson model is the one that best represents the adsorption isotherms of the corn kernels under the studied conditions. The values of the latent heat of vaporization of corn kernels, with water contents of 0.026 to 0.172 (d.b.), ranged from 3030.22 to $2567.92 \mathrm{~kJ} \mathrm{~kg}^{-1}$. The increase of moisture in the grains causes lower values of latent heat of vaporization.
\end{abstract}

Additional keywords: energy; moisture content; water; Zea mays.

\section{Introdução}

O milho é uma espécie originária da América Central, cultivado em grande parte do mundo, tem grande importância econômica devido às diversas formas de utilização, desde a alimentação animal até a indústria de alta tecnologia. O Brasil é o terceiro maior produtor de milho, ficando atrás apenas dos Estados Unidos e da China (Duarte; Kappes, 2015). Segundo a Companhia Nacional de Abastecimento (CONAB), a estimativa de produção de grãos de milho foi de aproximadamente $5.358 \mathrm{~kg} / \mathrm{ha}$ na safra 2017/18 (CONAB, 2018). Dessa forma, o país poderá se consolidar como importante exportador desse cereal no decorrer dos anos. Todavia, problemas relacionados à qualidade do grão podem comprometer a consolidação do Brasil como um grande fornecedor de milho.

As isotermas, ou curva de equilíbrio higroscópico, consistem em modelos matemáticos que são capazes de expressar a relação entre a umidade do grão e a atividade de água para uma determinada temperatura (Sousa et al., 2016; Goneli et al., 2014). Muitos autores estudaram as isotermas de diversos produtos: Teixeira et al. (2015), com higroscopicidade e calor isostérico do bagaço de cana-de-açúcar; Smaniotto et al. (2012), com isotermas de dessorção de grãos de milho.

Quando a umidade do grão entra em equilíbrio com a umidade do ar ambiente ocorre o fenômeno 
denominado equilíbrio higroscópico. As informações relacionadas ao equilíbrio higroscópico podem ser utilizadas diretamente nos processos de secagem e armazenamento (Hessini et al., 2015).

De acordo com Corrêa \& Almeida (1999), a partir das curvas de equilíbrio higroscópio, é possível determinar o calor latente de vaporização da água contida no interior dos grãos. O Calor latente de vaporização pode ser definido como a quantidade de energia requerida para remover a água de um produto em dessorção (Rodriguez-Arias et al., 1963; Corrêa et al., 1998) ou a energia liberada pelo produto durante a adsorção de água (Wang \& Brennan, 1991).

Este trabalho teve o objetivo de determinar e modelar as isotermas de adsorção dos grãos de milho, utilizando-se o método estático gravimétrico, nas temperaturas de 40,50 , e $60^{\circ} \mathrm{C}$, como também obter o calor latente de vaporização.

\section{Material e métodos}

O projeto foi desenvolvido no Laboratório de Química Instrumental, do Instituto Federal Goiano Campus Ceres, na cidade de Ceres, estado de Goiás. Para a determinação das isotermas de adsorção foi utilizado o método estático gravimétrico que utiliza soluções saturadas de sais, cujas atividades de água submetidas nas temperaturas de 40,50 e $60^{\circ} \mathrm{C}$ estão apresentadas na Tabela 1.

Tabela 1 - Atividade de água (decimal) de diversas soluções salinas nas temperaturas de 40, 50 e 60 ㄷ. Water activity (decimal) of several saline solutions at temperatures of 40,50 and $60^{\circ} \mathrm{C}$.

\begin{tabular}{|c|c|c|c|c|c|}
\hline \multirow{2}{*}{ Temperatura $\left(\mathrm{C}^{\circ}\right)$} & \multicolumn{5}{|c|}{ Sal } \\
\hline & $\mathrm{LiCl}$ & $\mathrm{MgCl}_{2}$ & $\mathrm{Mg}\left(\mathrm{NO}_{3}\right)_{2}$ & $\mathrm{NaCl}$ & $\mathrm{KCl}$ \\
\hline 40 & $0,11^{* *}$ & $0,31^{*}$ & $0,585^{\star *}$ & $0,74^{*}$ & $0,81^{*}$ \\
\hline 50 & $0,11^{* *}$ & $0,31^{*}$ & $0,585^{\star *}$ & $0,71^{*}$ & $0,77^{*}$ \\
\hline 60 & $0,11^{* *}$ & $0,295^{*}$ & $0,585^{\star *}$ & $0,70^{*}$ & $0,75^{\star}$ \\
\hline
\end{tabular}

** (Fiorentin et al,. 2010), * (Labuza et al., 1985)

As amostras dos grãos de milho da cultivar Hib s1 Truck TL TG VIP3 SYN7316 foram adquiridas na Fazenda Santa Bárbara, município de Santana do Araguaia-PA. Na realização do experimento, foram separados $300 \mathrm{~g}$ de grãos de milho para cada temperatura com umidade de $10 \%$ base seca (b.s.). Antes do processo de adsorção, os grãos de milho foram submetidos à estufa de ventilação forçada a $50{ }^{\circ} \mathrm{C}$ durante 72 horas, resultando em umidade de $5 \%$ em base seca (b.s.).

Em seguida, as soluções salinas preparadas foram depositadas em recipientes de vidro herméticos com volume de $1500 \mathrm{ml}$, contendo, em seu interior, suportes para três recipientes de sorção. Em cada recipiente de sorção, foram colocados $2 \mathrm{~g}$ de amostra, de modo que a superfície externa dos grãos ficasse em contato com o ar ambiente de sorção. Os recipientes em triplicata, contendo as amostras, foram colocados em câmaras tipo BOD (Biochemical oxygen demand), com temperaturas controladas de 40,50 e $60^{\circ} \mathrm{C}$. Os recipientes de sorção foram pesados em balança analítica com quatro casas decimais e com intervalos de $24 \mathrm{~h}$, até atingirem a umidade de equilíbrio higroscópico.

Posteriormente, o teor de água dos grãos do milho foi determinado conforme ASABE (2010). Os modelos matemáticos, frequentemente utilizados para representação da higroscopicidade de grãos, foram ajustados aos dados experimentais do milho, cujas expressões estão apresentadas na Tabela 2.

Tabela 2 - Modelos matemáticos utilizados para ajuste dos dados experimentais. Mathematical models used to fit the experimental data.

\begin{tabular}{|c|c|c|}
\hline Descrição & Modelo & \\
\hline Peleg & $\mathrm{Ue}=K_{1} \times(\mathrm{Aw})^{\mathrm{n}_{1}}+\mathrm{K} 2 \times(\mathrm{Aw})^{n_{2}}$ & (1) \\
\hline GAB & $U e=\left(\frac{X m \times c \times k \times A w}{(1-K \times A w) \cdot(1-k \times A w+c \times k \times A w)}\right)$ & (2) \\
\hline Henderson & $U e=\left[\frac{\ln (1-A w)}{(-k T)}\right]^{\frac{1}{b}}$ & (3) \\
\hline Henderson Modificado & $U e=\left[\frac{\ln (1-A w)}{(-k(T+c)}\right]^{\frac{1}{n}}$ & (4) \\
\hline Oswin & $\mathrm{Ue}=\mathrm{a}\left(\frac{\mathrm{Aw}}{1-A w}\right)^{\mathrm{b}}$ & (5) \\
\hline Halsey Modificado & $U e=\exp \left[\exp (a+b T) A w^{-c}\right]$ & (6) \\
\hline BET & $U e=\left(\frac{(X m \times c \times A w) \times\left(1-(n+1) A w^{n+1}\right.}{(1-A w) \times\left(1+(C-1) U R-C \times A w^{n+1}\right.}\right)$ & (7) \\
\hline
\end{tabular}

Ue - umidade de equilíbrio em \% b.s.; Aw - atividade de água (decimal); $\mathrm{T}$ - temperatura em ${ }^{\circ} \mathrm{C} ; \mathrm{a}, \mathrm{b}, \mathrm{c}, \mathrm{k}, \mathrm{Xm} \mathrm{e}$ $\mathrm{n}, \mathrm{n}_{1}, \mathrm{n}_{2}, \mathrm{~K} 1$ e K2 - parâmetros de ajuste dos modelos. 
Para seleção do melhor modelo matemático ajustado aos dados experimentais, foi utilizado o coeficiente de determinação $\left(R^{2}\right)$, o erro médio relativo $(P)$ (equação 8) e o erro médio estimado (SE) (equação 9). Além disso, foi utilizado a distribuição dos resíduos. A análise do comportamento dos resíduos foi considerada aleatória, quando for observada distribuição uniforme e mais próxima do eixo dos valores dos resíduos estimados durante o processo de adsorção.

$P=\frac{100}{n} \sum_{i=1}^{n}\left(\frac{|Y-\hat{Y}|}{Y}\right)$

$S E=\sqrt{\frac{\sum_{i=1}^{n}(Y-\widehat{Y})^{2}}{G L R}}$

Em que: $\mathrm{P}$ - erro médio relativo em \%; SE - erro médio estimado (decimal); $n=$ número de observações experimentais; $Y=$ valor observado experimentalmente; $\hat{Y}=$ valor estimado pelo modelo; $G L R=$ graus de liberdade do modelo.

A partir dos estudos de Clausius-Clapeyron, Othmer (1940) propôs a equação (10) para quantificar a pressão parcial do vapor contida em sistemas porosos:

$\operatorname{Ln}(P v)=\frac{L}{L^{\prime}} \operatorname{Ln}(P v s)+C$

Em que: Pvs: - pressão de vapor de saturação da água livre, para determinada temperatura $(\mathrm{T})$, de equilíbrio; Pv - pressão de vapor da água livre, para determinada temperatura $\mathrm{T}$, de equilíbrio; $\mathrm{L}$ : calor latente de vaporização da água do produto, $\mathrm{kJ} \mathrm{kg}^{-1}$; L': calor latente de vaporização da água livre, a temperatura de equilíbrio, $\mathrm{kJ} \mathrm{kg}^{-1}$; C: constante de integração.

Com o intuito de quantificar os valores da relação calor latente da água no grão e o calor latente de vaporização da água livre (LL'-1) para os grãos de milho, foi utilizada a metodologia proposta por Stefanini \& Roa (1980).

Ajustou-se a equação para a entalpia de vaporização da água, apresentada por Rodrigues-Arias (Brooker et al., 1992), com a inclusão do parâmetro "m" na equação (11), considerando a significância dos parâmetros a $1 \%$ de probabilidade pelo test $t$, para assim melhorar as estimativas de LL'-1 (Corrêa et al., 1998):

$\frac{L}{L^{\prime}}-1=\operatorname{axexp}\left(-b U e^{m}\right)$

Em que: L: calor latente de vaporização da água do produto, $\mathrm{kJ} \mathrm{kg}^{-1}$; L': calor latente de vaporização da água livre, a temperatura de equilíbrio, $\mathrm{kJ} \mathrm{kg}^{-1}$; a, b e m: coeficientes empíricos de ajuste do modelo; Ue: umidade de equilíbrio.

O calor latente de vaporização da água livre ( $L$ '; $\mathrm{kJ} \mathrm{kg}^{-1}$ ) à temperatura de equilíbrio $\left({ }^{\circ} \mathrm{C}\right)$ foi calculado utilizando-se a temperatura média (T) na faixa em estudo, em ${ }^{\circ} \mathrm{C}$, por meio da seguinte equação:

$L^{\prime}=2502,2-2,39 T$
Para o cálculo da pressão de vapor de saturação da água livre, Pvs, utilizou-se a equação de Tétens:

PVs $=0,61078 \times 10^{((7,5 . T) /(273,3+T))}$

O valor da pressão de vapor, Pv, foi determinado de acordo com a seguinte equação:

$\mathrm{Pv}=\mathrm{Aw} \times \mathrm{Pvs}$

Com o intuito de quantificar o calor latente de vaporização da água dos grãos de milho, em cada temperatura estudada, as equações 11 e 12 foram combinadas, originando a equação (15):

$L=(2502,2-2,39 T) \times\left[1+a \times \exp \left(-b U e^{m}\right)\right]$

\section{Resultados e discussão}

Na Tabela 3 estão apresentados os valores dos coeficientes das equações ajustadas aos dados experimentais, $\mathrm{R}^{2}, \mathrm{P}$ e $\mathrm{SE}$ dos modelos para isotermas de adsorção nas temperaturas de 40, 50 e $60 \stackrel{\circ}{\circ}$. Percebe que, para todos os dados experimentais ajustados, os coeficientes de determinação $\left(R^{2}\right)$ foram superiores a $90 \%$. Em geral, foi observado que os modelos Henderson Modificado e Oswin apresentaram maiores valores de $\mathrm{R}^{2}$ com valor de 97,12 e $96,20 \%$, respectivamente. Todavia, de acordo com Madamba et al. (1996), o coeficiente de determinação $\left(R^{2}\right)$ não constitui, sozinho, um bom critério para a seleção de modelos não lineares. Assim, os valores obtidos do erro médio estimado (SE), erro relativo $(P)$ e a distribuição de resíduos foram considerados.

De acordo com Siqueira et al. (2012), quanto menores os valores de SE melhor o ajuste do modelo aos dados observados. Verifica-se que dentre os modelos ajustados, os de Henderson Modificado e Oswin foram os que apresentaram menores valores de SE quando comparados aos demais, sendo os valores de 0,0402 e 0,0474, respectivamente.

Em relação à distribuição de resíduos, verificase que o modelo de Henderson Modificado e Halsey Modificado apresentaram distribuição aleatória (Tabela 2) e os modelos BET e Oswin, distribuição tendenciosa. $\mathrm{Na}$ figura 1 é mostrada a distribuição de resíduos de acordo com os modelos de Henderson Modificado (A) e de BET (B). É possível verificar que o modelo de Henderson Modificado (Figura 1A) apresenta a distribuição dos resíduos mais próximos ao eixo dos valores estimados do que comparado ao modelo de BET (Figura 1B). Dessa forma, esse critério reforça a seleção do modelo de Henderson Modificado como o mais indicado para representar o processo de adsorção de água para os grãos de milho.

Nota-se, na Tabela 3, que somente o modelo de Henderson Modificado apresentou o valor do erro médio relativo $(P)$ menor que 10\%. Dessa forma, recomenda-se esse modelo para a representação da higroscopicidade dos grãos de milho nas temperaturas estudadas. 
Tabela 3 - Parâmetros ajustados dos modelos de equilíbrio higroscópico e critérios estatísticos, para os grãos de milho submetidos ao processo de adsorção, nas temperaturas de 40,50 e $60 \stackrel{\circ}{ } \mathrm{C}$. Adjusted parameters of the hygroscopic equilibrium models and statistical criteria, for maize grains submitted to the adsorption process, at temperatures of 40,50 and $60 \stackrel{\circ}{C}$.

\begin{tabular}{llllll}
\hline Modelo matemático & \multicolumn{1}{c}{ Parâmetros } & $\mathrm{R}^{2}(\%)$ & $\mathrm{SE}($ decimal) & $\mathrm{P}(\%)$ & Distribuição \\
\hline Peleg & $\begin{array}{l}\mathrm{k} 1=333,6176^{\mathrm{ns}} \\
\mathrm{n} 1=48,7242^{\mathrm{ns}} \\
\mathrm{k} 2=0,1737^{*}\end{array}$ & 95,13 & 0,0561 & 14,17 & $\mathrm{~T}$ \\
$\mathrm{n} 2=0,8921^{*}$ & & & & \\
\hline GAB & $\begin{array}{l}\mathrm{X}=0,06765^{*} \\
\mathrm{C}=7,0405^{*} \\
\mathrm{k}=0,7527^{*}\end{array}$ & 94,71 & 0,0595 & 12,67 & $\mathrm{~T}$ \\
\hline Henderson & $\begin{array}{l}\mathrm{k}=0,0879^{*} \\
\mathrm{~b}=1,5357^{*}\end{array}$ & 95,94 & 0,0515 & 11,58 & $\mathrm{~T}$ \\
\hline Henderson Modificado & $\begin{array}{l}\mathrm{k}=0,3919^{*} \\
\mathrm{c}=31,2355^{\mathrm{ns}} \\
\mathrm{n}=1,5824^{*}\end{array}$ & 97,12 & 0,0402 & 9,90 & $\mathrm{~A}$ \\
\hline Oswin & $\begin{array}{l}\mathrm{a}=0,0854^{*} \\
\mathrm{~b}=0,4429^{*}\end{array}$ & 96,20 & 0,0474 & 11,90 & $\mathrm{~T}$ \\
\hline Halsey Modificado & $\begin{array}{l}\mathrm{a}=-4,1792^{*} \\
\mathrm{~b}=0,0108^{*} \\
\mathrm{c}=1,7248^{*}\end{array}$ & 94,18 & 0,0612 & 14,06 & $\mathrm{~A}$ \\
\hline BET & $\begin{array}{l}\mathrm{Xm}=207,1060 \text { ns } \\
\mathrm{c}=0,0009^{\mathrm{ns}} \\
\mathrm{n}=1,0003^{\mathrm{ns}}\end{array}$ & 93,60 & 0,0662 & 15,85 & $\mathrm{~T}$ \\
\hline
\end{tabular}

*Significativo a 5\% de probabilidade pelo teste t. T: Distribuição tendenciosa dos resíduos; A: Distribuição aleatória dos resíduos; ns não significativo.
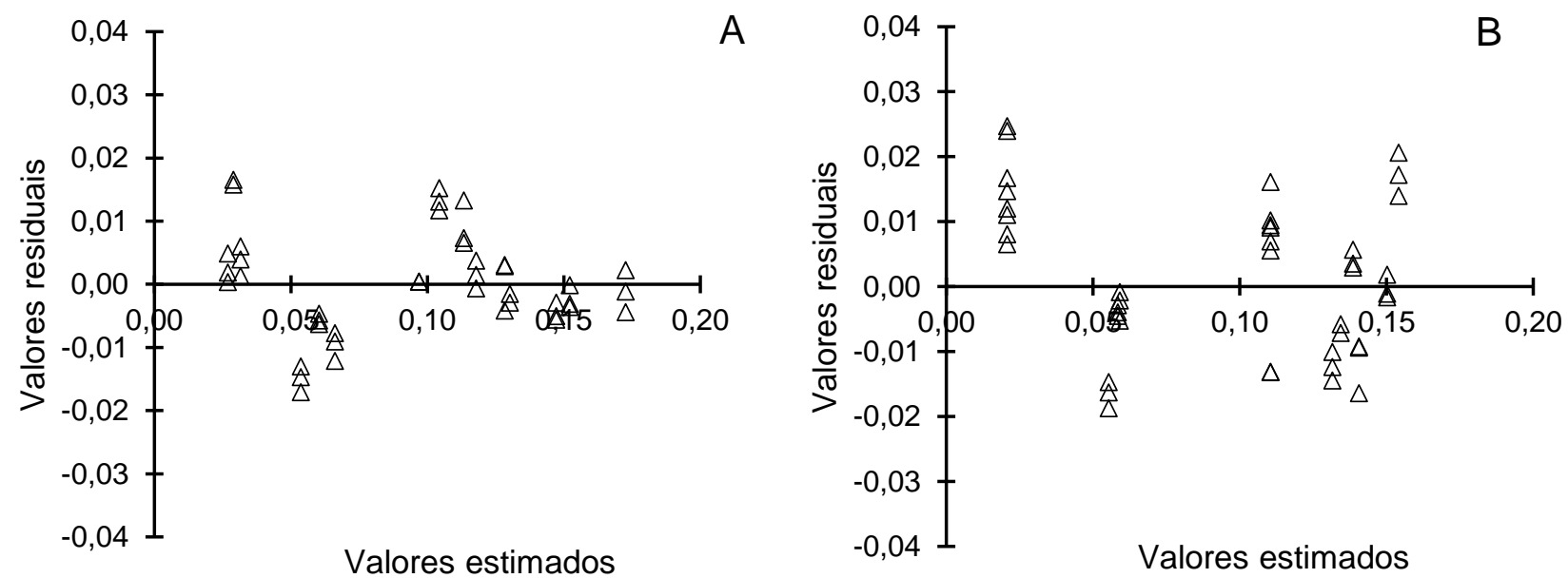

Figura 1- Comportamento da distribuição aleatória dos resíduos pelo modelo Henderson Modificado (A) e comportamento da distribuição tendenciosa dos resíduos pelo modelo BET (B). Behavior of random waste distribution by the Modified Henderson model ( $A$ ) and behavior of biased waste distribution by the BET model (B).

Os resultados encontrados neste trabalho se assemelham com os encontrados por Teixeira et al. (2015), que trabalharam com equilíbrio higroscópico do bagaço de cana-de-açúcar; Bracht et al. (2015), com isotermas de equilíbrio das sementes de uva das variedades cabernet sauvignon e bordô; e Corrêa et al. (2005), com equilíbrio higroscópico das espigas de milho, em que Henderson Modificado foi um dos modelos que representou de forma satisfatória a higros- copicidade dos dados amostrais.

As isotermas de adsorção dos grãos de milho são mostradas na Figura 2. Verifica-se que elas possuem formato sigmoidal, características de curvas do tipo II, de acordo com a classificação de Brunauer et al. (1938), consideradas o padrão para produtos agrícolas. Nota-se a influência da temperatura sobre a higroscopicidade nos grãos de milho, que está de acordo com o observado na maioria dos produtos 
agrícolas. Para se atingir o mesmo teor de água de equilíbrio, o aumento da temperatura requer o aumento da atividade de água ou, de forma semelhante, para a atividade de água, o teor de água de equilíbrio diminui com o aumento da temperatura (Corrêa et al., 2014).

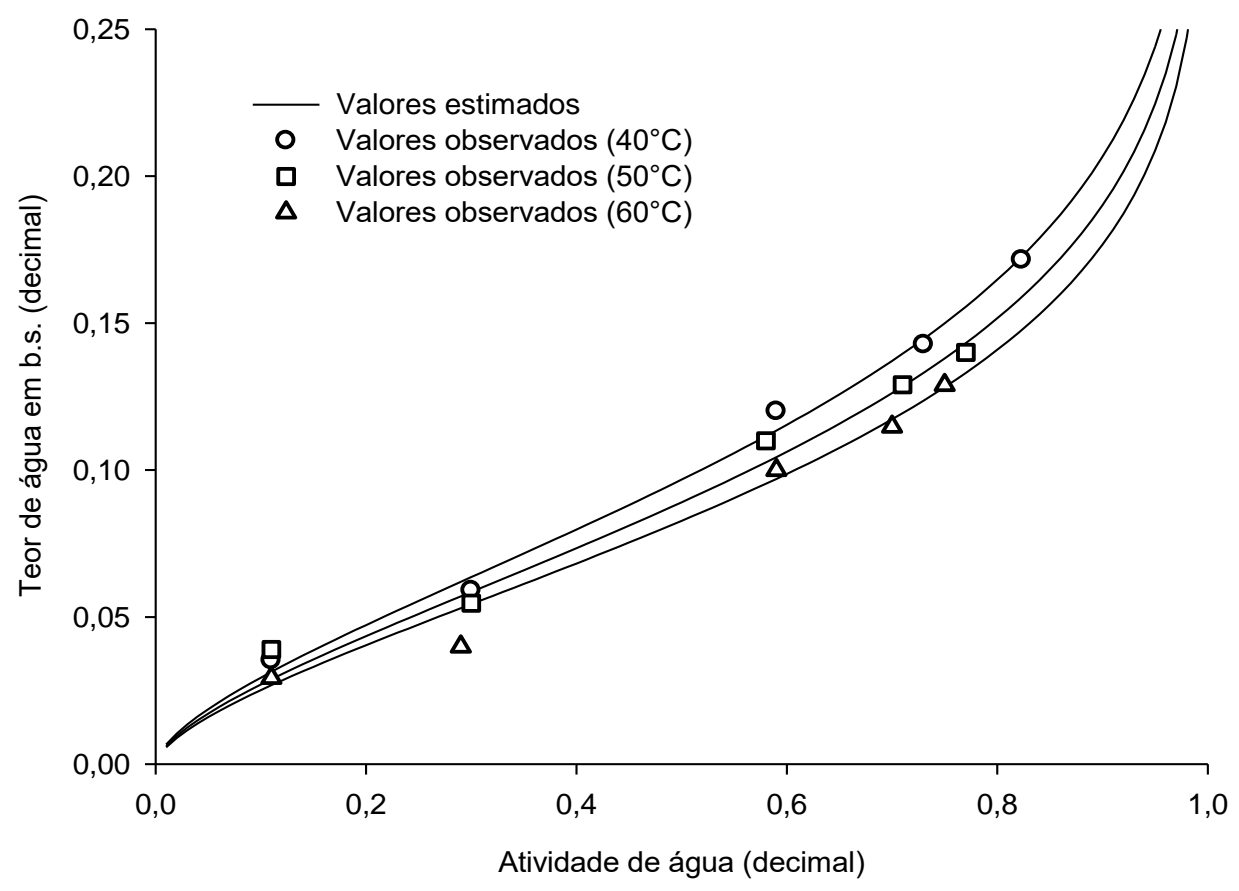

Figura 2 - Isotermas de adsorção dos grãos de milho, conforme o modelo de Henderson Modificado. Adsorption isotherms of corn grains, according to the Modified Henderson model.

A figura 3 mostra os valores experimentais e estimados oriundos da relação do calor latente de vaporização dos grãos de milho com o calor latente de vaporização da água livre (LL'-1) em função do teor de água dos grãos. Percebe-se que a relação $L^{2} L^{-1}$ é inversamente proporcional ao teor de água dos grãos de milho, ou seja, quanto maior essa relação, menor será o teor de água nos grãos. Verifica-se que todos os valores obtidos para a relação LL'-1 foram maiores que 1. Isso significa que a energia liberada pelos grãos para absorver água durante o processo de adsorção, é sempre maior que a energia necessária para evaporar a água pura. Comportamentos similares foram observados em grãos milho, cultivar AG 7088, por Smaniotto et al (2012), e com grãos de café e soja, por Stefanini \& Roa (1980). 


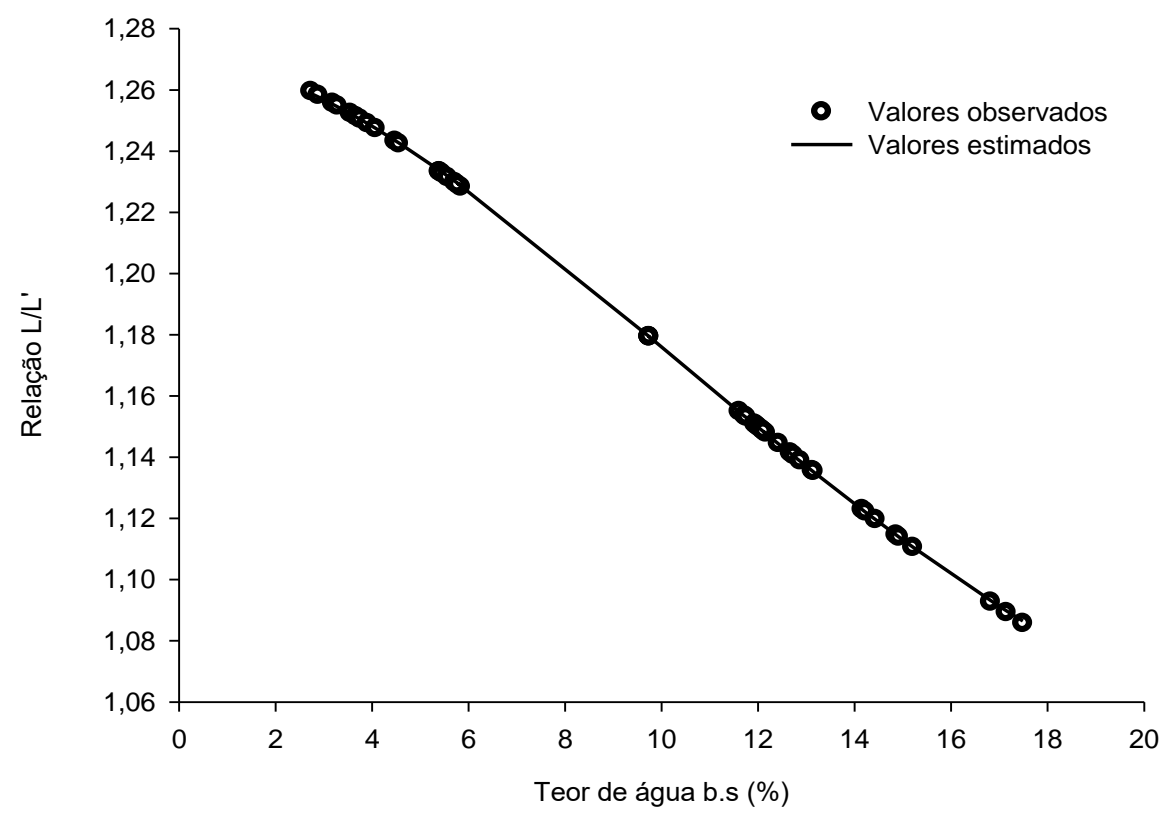

Figura 3 - Valores da relação LL'-1 experimentais e observados em função dos teores de água de equilíbrio dos grãos de milho. Values of the experimental $L L^{11}$ ratio and observed as a function of the equilibrium water content of the corn grains.

$\mathrm{Na}$ Tabela 4, encontram-se os valores dos parâmetros "a", "b" e "m" para a equação 11. Esses coeficientes são utilizados para calcular a relação LL'-1. Verifica-se, na Tabela 3, que a equação 11 foi devidamente ajustada para o cálculo da relação $L^{\prime \prime-1}$, na qual foi observado alto valor do coeficiente de determinação $\left(R^{2}=0,9999\right)$, e todos os parâmetros foram significativos a $1 \%$ pelo test t. Portanto, a equação pode ser utilizada para determinar o calor latente de vaporização para os grãos de milho.

Tabela 4 - Coeficientes utilizados para o cálculo da relação LL'-1 dos grãos de milho.

\begin{tabular}{cccc}
\hline$a$ & $b$ & $m$ & $R^{2}$ \\
\hline 0,2707 & 23,8919 & 1,7439 & 0,9999 \\
\hline
\end{tabular}

** Significante a $1 \%$ de probabilidade pelo test $\mathrm{t}$.

Substituindo-se os valores de "a", "b" e "m" da Tabela 4, na equação proposta por Corrêa et al (1998), e adicionando o calor latente de vaporização da água livre, obteve-se a equação que pode ser utilizada para o cálculo do calor latente de vaporização dos grãos de milho para cada temperatura.

São mostrados, na figura 4 , os valores obtidos do calor latente de vaporização da água para os grãos de milho, bem como as curvas estimadas pelo ajuste da equação 16 , em função do teor de água nas temperaturas de 40,50 e $60^{\circ} \mathrm{C}$. Os valores do calor latente de vaporização dos grãos de milho, com o teor de água em torno de $2,69 \%$ a $17,26 \%$ (b.s), variaram de 3030,22 a $2567,92 \mathrm{~kJ} \mathrm{~kg}^{-1}$. Smaniotto et al (2012), que trabalharam com grãos de milho cultivar AG7088, encontraram valores semelhantes de 2775, 87 a $2468,14 \mathrm{~kJ} \mathrm{~kg}^{-1}$. Segundo Corrêa \& Almeida (1999), para grãos e sementes, o calor latente de vaporização consiste na quantidade de energia necessária para evaporar uma unidade de massa de água. Trata-se de uma propriedade física de fundamental importância na composição dos modelos matemáticos de secagem. 


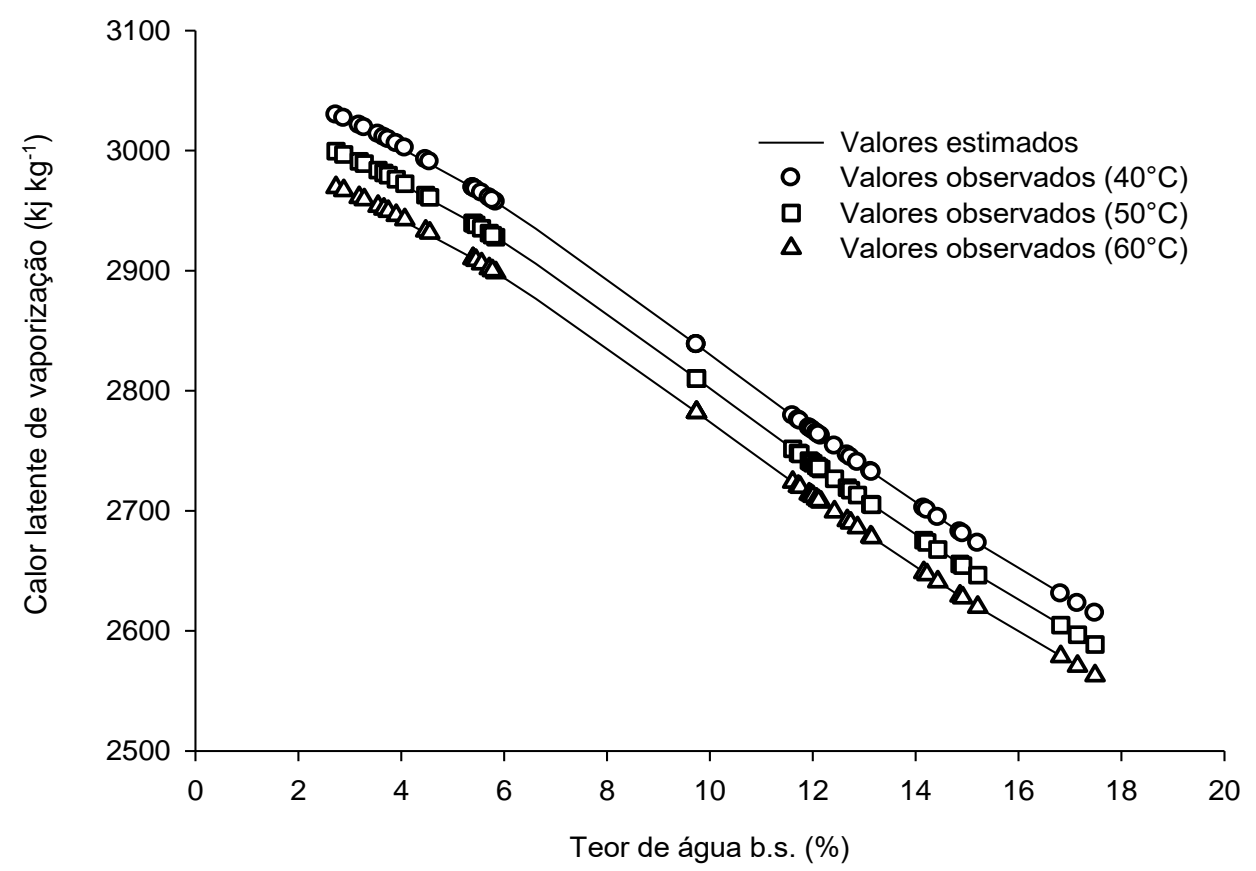

Figura 4 - Calor latente de vaporização da água para os grãos de milho nas diferentes temperaturas estudadas. Latent heat of water vaporization for corn kernels at the different temperatures studied.

Verifica-se, ainda, na figura 4, que os valores do calor latente de vaporização dos grãos de milho aumentam com a redução do teor de água dos grãos. Esse comportamento também foi observado por Smaniotto et al. (2012), que trabalharam com grãos de milho cultivar AG7088.

\section{Conclusões}

O modelo de Henderson Modificado pode ser utilizado para representar o equilíbrio higroscópico dos grãos de milho nas temperaturas de 40, 50 e $60 \stackrel{\circ}{\circ}$.

A partir da redução do teor de água, há aumento na energia liberada pelos grãos durante o processo de adsorção da água, e os valores do calor latente de vaporização dos grãos de milho, com teores de água de 0,026 a 0,172 (b.s), reduziram de 3030,22 para $2567,92 \mathrm{~kJ} \mathrm{~kg}^{-1}$.

\section{Agradecimentos}

Esta pesquisa foi apoiada pelo Instituto Federal Goiano (Campus Ceres), Fundação de Amparo à Pesquisa do Estado de Goiás (FAPEG) e o Conselho Nacional de Desenvolvimento Científico e Tecnológico (CNPq).

\section{Referências}

ASABE (2010) Moisture Measurement - Forages: Standard S358.2 DEC1988, R2008. In: American Society of Agricultural and Biological Engineers (Ed.). Standards, Engineering Practices, and Data. St. Joseph: ASABE, 684-685p.
Bracht, CK, Menezes MLD, Ugri MCBA, Pareira NC (2015) Determinação das isotermas de equilíbrio das sementes de uva das variedades Cabernet Sauvignon e Bordô. Engevista, Niterói 17(1): 44-58.

Brooker DB, Bakker-Arkema FW, Hall CW (1992) Drying and storage of grains and oilseeds. Westport: AVI Publishing Company. 450p.

Brunauer S, Emmett PH, Teller E (1938) Adsorption of gases in multimolecular layer. Journal of American Chemistry Society 60 (1): 309-312.

CONAB (2018) Acompanhamento de safra brasileira: grãos, safra 2017/18, quinto levantamento. Brasília.

Corrêa PC, Almeida FDAC (1999) Comparação de modelos matemáticos de equilíbrio higroscópico para semente e fibra de algodão herbáceo, cultivar redenção. Revista de Oleaginosas e Fibrosas 3(1):1-6.

Corrêa PC, Botelho, FM, Botelho SDCC, Goneli ALD (2014) Isotermas de sorção de água de frutos de Coffea canéfora. Revista Brasileira de Engenharia Agrícola e Ambiental 18(10): 1047-1052.

Corrêa PC, Christ D, Martins JH, Mantovani BHM (1998) Curvas de dessorção e calor latente de vaporização para as sementes de milho pipoca (Zea mays). Revista Brasileira de Engenharia Agrícola e Ambiental 2(1):7-11.

Corrêa PC, Resende O, Ribeiro DM (2005) isotermas de sorção das espigas de milho: obtenção e modelagem. Revista Brasileira de Milho e Sorgo 4(1): 126-134. 
Duarte AP, Kappes C (2015) Evolução dos Sistemas de Cultivo de Milho no Brasil. Informações Agronômicas (brasil) 152(1): 15-18.

Fiorentin LD, Menon BT, Barros STD, Pereira NC, Lima OCDM, Modenes AN (2010) Isotermas de sorção do resíduo agroindustrial bagaço de laranja. Revista Brasileira de Engenharia Agrícola e Ambiental 14(6): 653-659.

Goneli ALD, Nasu AK, Gancedo R, Araújo WD, Sarath KLL (2014) Cinética de secagem de folhas de erva baleeira (Cordia verbenacea DC.). Revista Brasileira de Plantas Medicinais. 16(1):434-443.

Hessini L., Bettaieb E, Desmorieux H, Torres SS, Touil A (2015) Desorption isotherms and thermodynamic properties of prickly pear seeds. Industrial Crops and Products 67(1): 457-465 http://dx.doi.org/10.1016/j.indcrop.2015.01.078.

Labuza TP, Kaauane A, Chen JY (1985) Effect of temperature on the moisture sorpion isotherms and water activity shift of two dehydrated foods. Journal of Food Science 50(1): 385-391.

Madamba PS, Driscoll RH, Buckle KA (1996) The Thinlayer Drying Characteristics of Garlic Slices. Journal of Food Engineering. 29(1): 75-97 doi:htpp://dx.doi.org/10.1016/0260- 774(95)00062-3>

Othmer DF (1940) Correlation vapor pressure and latent heat data: A new plot. Industrial and Engineering Chemistry, Washington, 32(6).
Rodriguez-Arias JH, Hall CW, Bakker-Arkema FW (1963) Heat of vaporization for shelled corn. Agricultural Engineering 40:677-683.

Siqueira VC, Resende O, Chaves TH (2012) Drying Kinetics of Jatropha seeds. Revista Ceres. 59(1): 171177 doi: http://dx.doixorg/10.1590/S003473X20100200004

Smaniotto TAS, Resende O, Oliveira DEC, Sousa KA, Campos RC (2012) Isotermas e calor latente de dessorção dos grãos de milho da cultivar AG 7088. Revista Brasileira de Milho e Sorgo 11(3):312-322.

Sousa KAD, Resende O, Carvalho BDS (2016) Determination of desorption isotherms, latent heat and isosteric heat of pequi diaspore. Revista Brasileira de Engenharia Agrícola e Ambiental 20(5):493-498.

Stefanini R, Roa G (1980) Algorítmo geral para determinação do calor latente de vaporização da água dos produtos biológicos. Revista Brasileira de Armazenamento 5(1):30-42.

Teixeira LP, Andrade ETD, Espíndola RGP (2015) Determinação do equilíbrio higroscópico e do calor isostérico do bagaço de cana-de-açúcar. Engenharia Agrícola, 35(3): 555-566.

Wang N, Brennan JG (1991) Moisture sorption isotherms characteristics of potatoes at four temperatures. Journal of Food Engineering 14(1)269-287. 